\title{
ARTIGo Técnico \\ INTEGRAÇÃO DE PROCESSOS FíSICO-QUÍMICOS E OXIDATIVOS AVANÇADOS PARA REMEDIAÇÃO DE PERCOLADO DE ATERRO SANITÁRIO (CHORUME)
}

\author{
INTEGRATION OF PHYSICAL CHEMISTRY AND ADVANCED OXIDATIVE \\ PROCESSES FOR REMEDIATION OF LANDFILL LEACHATE
}

JAILSON RODRIGO PACHECO

Licenciado em Química e Mestre em Química Analítica pela UFPR

Patricio G. Peralta-Zamora

Químico laboratorista pela Universidad De Chile, Mestre em Química Analítica e Doutor em Ciências, Unicamp. Professor Adjunto, DQ-UFPR

Recebido: 05/03/04 Aceito: 04/08/04

\section{RESUMO}

Neste trabalho está sendo proposto um processo integrado para a remediação de chorume, consistente na separação preliminar das substâncias húmicas, por precipitação em meio ácido, e o tratamento subseqüente do sobrenadante por meio de processos oxidativos avançados. Em geral, a precipitação preliminar permite remover uma pequena parcela da DQO (14\%) e grande parte da cor, o que permite significativas melhoras no tratamento fotoquímico posterior. Quando aplicado na forma de um sistema de múltiplas adições de peróxido de hidrogênio, o sistema $\mathrm{UV} / \mathrm{H}_{2} \mathrm{O}_{2}$ permite descolorações da ordem de $95 \%$ e reduções de DQO de 75\%, em tempos de reação de $120 \mathrm{~min}$. Trata-se de um resultado bastante promissor, principalmente levando-se em consideração a elevada resistência da matriz em estudo.

PALAVRAS-CHAVE: Chorume, processos integrados, processos oxidativos avançados.

\begin{abstract}
In this work, we propose the integration of processes for remediation of landfill leachate, where humic substances are preliminary precipited and separated in acidic conditions and an advanced oxidative process further treats the soluble fraction. Typically, the precipitation of humic substances reduces $14 \%$ of the COD content and a great part of the intrinsic color, fact that significantly improves the efficiency of the photochemical treatment. When applied as a successive $\mathrm{H}_{2} \mathrm{O}_{2}$ additions system the $\mathrm{UV} / \mathrm{H}_{2} \mathrm{O}_{2}$ process induce color and $\mathrm{COD}$ reductions of 95 and $75 \%$ respectively, in reaction times of 120 min. This results are very promissory, principally because the recalcitrant character of the leachate matrix.
\end{abstract}

KEYWORDS: Landfill leachate, integrate process, advanced oxidative process.

\section{INTRODUÇÃO}

Estima-se que cada brasileiro produza $1,3 \mathrm{~kg}$ de lixo por dia, o que representa a geração diária, no Brasil, de aproximadamente 230 mil toneladas. A disposição final deste lixo varia conforme a região onde é coletado, mas em média, cerca de $71 \%$ é destinado a aterros (sanitários ou controlados) e $26 \%$ a lixões a céu aberto. Outras formas de disposição são bastante menos significativas (IBGE, 2003).

Após ser alocado em seu destino final, o lixo passará por processos físicos, químicos e biológicos de decomposição, produzindo frações residuais gasosas e líquidas (Lo, 1996). A fração líquida é resultado da decomposição do lixo, aliado a fatores ambientais relacionados com o regime pluviométrico, a temperatura do aterro e a composição dos resíduos depositados. A ação de bactérias acetogênicas e metanogênicas acelera a decomposição do lixo, enquanto que a percolação da água de chuva carrega os produtos de degradação para as camadas inferiores do aterro. Ao chegar ao aterro, o lixo passa por um processo de decomposição assistido por bactérias acetogênicas, o que dá um caráter ácido ao chorume produzido. Com o passar do tempo, há um aumento na concentração de bactérias metanogênicas, o que leva à produção de um chorume com características básicas, contendo espécies muito mais recalcitrantes do que as produzidas nas fases preliminares de decomposição. Em geral, estima-se que o principal problema ambiental associado a este tipo de percolado está representado pelos compostos orgânicos xenobióticos, grande parte dos quais apresenta toxicidade elevada (Christensen et al, 2001; Im et al, 2001; Lin e Chang, 2000).

Os processos convencionais utilizados no tratamento do chorume são baseados em processos fisico-químicos e biológicos. Os primeiros costumam ser fundamentados em processos de adsorção e em rotinas de precipitação-floculação, que apresentam elevada eficiência de depuração. Entretanto, as substâncias contaminantes não são degradadas, o que necessariamente implica a geração de fases sólidas (lodos) altamente contaminados (Bae et al, 1999). Processos biológicos, por sua vez, podem ser aplicados na forma de rotinas aeróbias, anaeróbias e facultativas (Freire et al, 2000; Stroot et al, 2001). Infelizmente, características 
como necessidade de longos tempos de residência (variando de dias até semanas) e baixa eficiência na remoção de compostos recalcitrantes e coloridos (Freire et al, 2000), fazem com que a sua eficiência seja bastante discutida.

Nos últimos anos, os processos oxidativos avançados (POAs) têm sido considerados como uma excelente alternativa para o tratamento de resíduos com características como as apresentadas pelo chorume. Isto é, volumes relativamente pequenos, elevada Demanda Química de Oxigênio (DQO), reduzida Demanda Bioquímica de Oxigênio (DBO) e presença de espécies coloridas, recalcitrantes e tóxicas. Estes processos são baseados na geração do radical hidroxila $(\cdot \mathrm{OH})$, um poderoso e altamente reativo agente oxidante $\left(\mathrm{E}^{0}=2,8 \mathrm{~V}\right)$, o qual pode promover a degradaçãao de inúmeros poluentes, em tempos bastante reduzidos (Camel e Bermond, 1998; Espulgas et al, 2002; Freire et al, 2000; Kunz et al, 2002).

Devido a sua alta reatividade, o radical hidroxila deve ser gerado in situ. Dentre as várias formas de geração, destaque para os processos homogêneos fundamentados na decomposição fotocatalisada de peróxido de hidrogênio (Leahy e Schreve, 2000; Crittenden, et al, 1999) e para os sistemas Fenton (Chamarro et al, 2001; Yoon et al, 1998) e foto-Fenton (Ghaly etal, 2001; Wu etal, 1999) (Equações 1-3, respectivamente).

$$
\begin{aligned}
& \mathrm{H}_{2} \mathrm{O}_{2}+\mathrm{hv}(254 \mathrm{~nm}) \rightarrow 2 \cdot \mathrm{OH} \\
& \mathrm{Fe}^{2+}+\mathrm{H}_{2} \mathrm{O}_{2} \rightarrow \mathrm{Fe}^{3+}+\mathrm{OH}^{-}+\mathrm{OH} \\
& \mathrm{Fe}^{3+}+\mathrm{H}_{2} \mathrm{O}+\mathrm{h} v(\mathrm{UV} \text { ou Vis }) \rightarrow \\
& \mathrm{Fe}^{2+}+\mathrm{H}^{+}+\mathrm{OH}
\end{aligned}
$$

Embora extremamente eficientes e inespecíficos, os POAs apresentam duas limitaçōes no tratamento de matrizes de chorume. Uma está representada pela elevada carga orgânica (DQO $\approx 5000 \mathrm{mg} \mathrm{O}_{2} \mathrm{~L}^{-1}$ ), que costuma diminuir drasticamente a eficiência dos processos, e a outra pela elevada concentração de ácidos húmicos, que absorvem fortemente na regiāo do ultravioleta-visível (Aguer et al, 1996), reduzindo significativamente a eficiência dos processos irradiados. Para contornar este problema, neste trabalho está se propondo a associação de processos oxidativos avançados com uma etapa preliminar de separação de substâncias húmicas. Conhecidamente, estes compostos precipitam em meio ácido, podendo ser separados por simples filtração (Nanny e Ratasuk, 2002).

\section{MATERIAIS E MÉTODOS}

\section{Reagentes}

Sulfato ferroso (ISOFAR, 99\% de pureza), ácido sulfúrico concentrado (QUIMIBRÁS) e Oxigênio grau comercial (WHITE MARTINS), foram utilizados como recebidos. Peróxido de hidrogênio (Peróxidos do Brasil, $58 \% \mathrm{~m} / \mathrm{m}$ ) foi padronizado por titulação permanganatométrica.

Amostras de chorume foram coletadas no Aterro da Cachimba, que atende à cidade de Curitiba e região metropolitana. Estas amostras correspondem a uma fração já tratada por processos biológicos convencionais, principalmente representados por uma seqüência de lagoas aeróbias e facultativas.

\section{Precipitação em meio ácido}

As substâncias húmicas foram precipitadas sob agitação magnética em $\mathrm{pH}$ próximo a 1,0, utilizando-se ácido sulfúrico $20 \%(\mathrm{~m} / \mathrm{m})$. O sólido foi separado por centrifugação seguida de filtração em membrana Milipore ${ }^{\circledR} 0,44 \mathrm{~mm}$.

\section{Tratamento fotoquímico}

Foi efetuado em reator fotoquímico clássico com capacidade de 120 mL (Figura 1), equipado com refrigeração por água, agitação magnética e sistema de oxigenação. A radiação ultravioleta é proporcionada por uma lâmpada a vapor de mercúrio de $125 \mathrm{~W}$ (sem o bulbo protetor), inserida na solução por meio de um bulbo de quartzo (radiação ultravioleta) ou de vidro Pyrex (radiação acima de $300 \mathrm{~nm}$ ). Oxigênio foi continuamente borbulhado, com vazão de aproximadamente $30 \mathrm{~mL} \mathrm{~min}^{-1}$.

\section{Controle analítico}

Medidas espectroscópicas na região do ultravioleta-visível foram realizadas em espectrofotômetro SCINCO-1100, utilizando-se cubetas de quartzo de $1,0 \mathrm{~cm}$. A resposta utilizada corresponde à área espectral, integrada entre 200 e $750 \mathrm{~nm}$.

A demanda química de oxigênio foi determinada de acordo com procedimento padrão, fundamentada em sistema de refluxo fechado e determinação espectrofotométrica (APHA, 1995).

Peróxido de hidrogênio residual foi determinado de acordo com procedimento adaptado de Oliveira et al (2001), baseado na reação entre peróxido de hidrogênio e o íon metavanadato $\left(\mathrm{VO}_{3}^{-}\right)$. A reação leva à formação de íon peroxovanadato $\left(\mathrm{VO}_{3}^{+}\right)$, que absorve fortemente em $446 \mathrm{~nm}$ (Oliveira et al, 2001).

Fenóis totais foram determinados de acordo com procedimento padrão (APHA, 1995), fundamentado na reação com reagente de Folin-Ciocalteau.

Espectros de fluorescência foram registrados em um espectrofotômetro HITACHI 4500, utilizando-se cubetas multifacetadas de quartzo.

A avaliação de toxicidade aguda foi realizada por bioensaio, baseado na determinação turbidimétrica da inibição do crescimento de culturas de Escherichia coli (Jardim et al, 1990).

\section{RESULTADOS E DISCUSSÃO}

\section{Caracterização da amostra de chorume}

O chorume apresenta uma composição extremamente variável, dependente, dentre outros fatores, das condições clímato-ambientais, da composição dos resíduos depositados e do manejo do aterro. No período de um ano, foram feitas quatro coletas de amostra, as quais apresentaram valores significativamente diferentes para todos os parâmetros analisados. Assim, as quatro amostras coletadas foram misturadas, dando origem à amostra de trabalho cujas características físicoquímicas são apresentada na Tabela 1 .

Esta composição média é característica de um chorume que se encontra em fase de decomposição metanogênica (idade superior a 5 anos), o que pode ser comprovado pelo seu elevado $\mathrm{pH}$ e reduzida DQO (Lo, 1996). No chorume formado em aterros antigos, a concentração de compostos orgânicos biodegradáveis é extremamente baixa, representando cerca de $10 \%$ da fração orgânica total. Além disto, grande parte da matéria orgânica presente corresponde a compostos húmicos e fúlvicos, reconhecidamente resistentes ao tratamento oxidativo avançado (Kang et al, 2002).

\section{Precipitação em meio ácido}

O sistema de precipitação por acidificação do meio até $\mathrm{pH}$ próximo de 1,0 é um processo eficiente para a remo- 


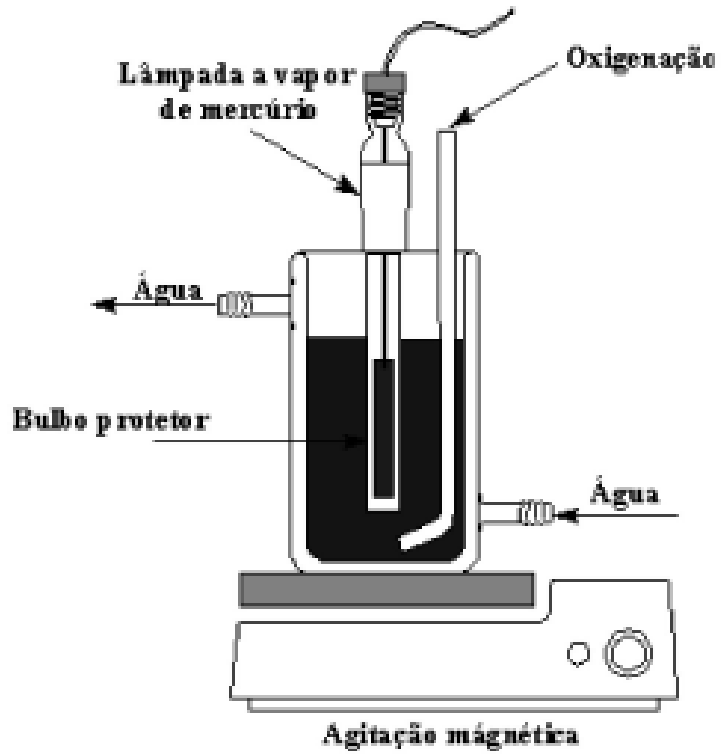

Figura I - Reator fotoquímico convencional (capacidade: $/ 20 \mathrm{ml}$ )

\begin{tabular}{cc}
\multicolumn{2}{c}{$\begin{array}{c}\text { Tabela I - Composição físico-química média das } \\
\text { amostras de chorume }\end{array}$} \\
\hline Parâmetro & Valor \\
\hline DQO & $4500 \mathrm{mg} \mathrm{O}_{2} / \mathrm{L}$ \\
Área Espectral & 658,7 u.a. \\
pH & 8,6 \\
Toxicidade (E. coli) & $25 \%$ de inibição \\
Ferro & $9,6 \mathrm{mg} / \mathrm{L}$ \\
Cloretos & $270,8 \mathrm{mg} / \mathrm{L}$ \\
Fração Precipitada Ácido & $3000 \mathrm{mg} / \mathrm{L}$ \\
\hline
\end{tabular}

a protonação dos grupos carboxílicos diminui bastante a sua solubilidade(Nanny e Ratasuki, 2002). Como pode ser observada na seqüência de espectros de fluorescência apresentada na Figura 2A, a comparação com uma substância húmica padrão permite verificar a efetiva precipitação das substâncias húmicas, assim como a sua posterior recuperação por dissolução em meio alcalino. Os espectros apresentados na Figura 2B, junto com os antecedentes sumarizados na Tabela 2 , sugerem que o processo de precipitação leva à separação de outras substâncias presente no chorume, como aminoácidos aromáticos, compostos policíclicos aromáticos e carboidratos.

Em média, o processo de precipitação gerou $3 \mathrm{~g}$ de sólidos por litro de chorume, o que contribuiu com uma diminuição de 50\% da área espectral e 14\% da DQO. Adicionalmente, a cor das amostras de chorume foi drasticamente reduzida, o que certamente contribui com a eficiência do processo fotoquímico posterior.

A geração de uma fração sólida talvez possa levantar alguns questionamentos. Entretanto, por se tratar de uma fase que contém essencialmente ácidos húmicos, a aplicação de um processo de floculação que permitisse a sua recuperação seria bastante justificável.

\section{Tratamento fotoquímico}

Estudos preliminares, envolvendo a degradação da fração líquida resultante do processo de precipitação ácida, foram realizados em reator convencional, utilizando-se condiçōes experimentais previamente otimizadas. Os resultados, sumarizados na Tabela 3 , correspondem a um tratamento de $60 \mathrm{~min}$. Com reduçôes de DQO e área espectral da ordem de $50 \%$ e $70 \%$, respectivamente, o siste$\mathrm{ma} \mathrm{UV} / \mathrm{H}_{2} \mathrm{O}_{2}$ é o que proporcionou melhores resultados. A baixa eficiência dos processos Fenton, principalmente no modo fotoquimicamente assistido, provavelmente esteja relacionada com a extrema acidez imposta pelo processo de precipitação preliminar ( $\mathrm{pH}$ 1). Nestas condiçōes, os íons ferrosos encontram-se na forma de hexaquo-complexos $\left(\left[\mathrm{Fe}\left(\mathrm{H}_{2} \mathrm{O}\right)_{6}\right]^{2+}\right)$, espécies fotoquimicamente menos ativa que o seu análogo tetraquo-dihidroxo $\left(\left[\mathrm{Fe}\left(\mathrm{H}_{2} \mathrm{O}\right)_{4}(\mathrm{OH})_{2}\right]\right)$, que prevalece em $\mathrm{pHs}$ próximos de 4,0 (Neyens e Bayens, 2003). Uma vez que o peróxido de hidrogênio foi completamente consumido em 60 min de tratamento, e em função da reação não se processar na sua ausência, um estudo envolvendo adiçōes sucessivas foi realizado. A estratégia consistiu em manter a concentração final entre 1500 e $1600 \mathrm{mg} \mathrm{L}^{-1}$ de $\mathrm{H}_{2} \mathrm{O}_{2}$, distribuindo de acordo com a sequiência apresentada na Tabela 4. Os resultados indicam que a melhor estratégia consiste em uma adição inicial de $1000 \mathrm{mg} \mathrm{L}^{-1}$, seguida de três adiçōes sucessivas de menor concentração $\left(200 \mathrm{mgL}^{-1}\right)$. Considerando os parâmetros físico-químicos iniciais para o chorume, estas condiçōes levaram a reduçōes de $\mathrm{DQO}$ e da área espectral da ordem $75 \%$ e $97 \%$, respectivamente, para tempos de reação de $120 \mathrm{~min}$.

Observando-se a seqüência de espectros apresentada na Figura 3, é possível verificar uma descoloração praticamente completa da matriz, assim como uma remoção bastante significativa de estruturas aromáticas, que absorvem fortemente na região compreendida entre 250 e $300 \mathrm{~nm}$. O sinal remanescente após 120 min de tratamento representa aproximadamente $25 \%$ do valor inicial da DQO, devendo corresponder principalmente a ácidos carboxílicos e, em menor grau, a estruturas fenólicas formadas no decorrer do processo (ver Figura 3B). Finalmente, é interessante salientar que o peróxido de hidrogênio e completamente consumido nos tempos praticados, $o$ que implica a não-inclusão de agentes químicos que aumentem o potencial poluente da matriz. 
Tabela 2 - Emissões máximas para a fração precipitada ácida recuperada por dissolução em meio básico

\begin{tabular}{ccc}
\hline $\begin{array}{c}\text { Comprimento de onda de } \\
\text { emissão máximo }(\mathrm{nm})\end{array}$ & Substância mais provável & Referência \\
\hline 292 & $\begin{array}{c}\text { Aminoácidos aromáticos e compostos } \\
\text { voláteis de cadeia alifática } \\
\text { altamente conjugada } \\
\text { Naftaleno e seus derivados }\end{array}$ & Peuravouri et al, 2002 \\
328 & Compostos policíclicos aromáticos et al, 2002 \\
347 & Carboidratos & Peuravouri et al, 2002 \\
386 & Ácidos Húmicos & Chen et al, 2003 \\
481 & & $*$
\end{tabular}

* Por comparação com padrão Aldrich
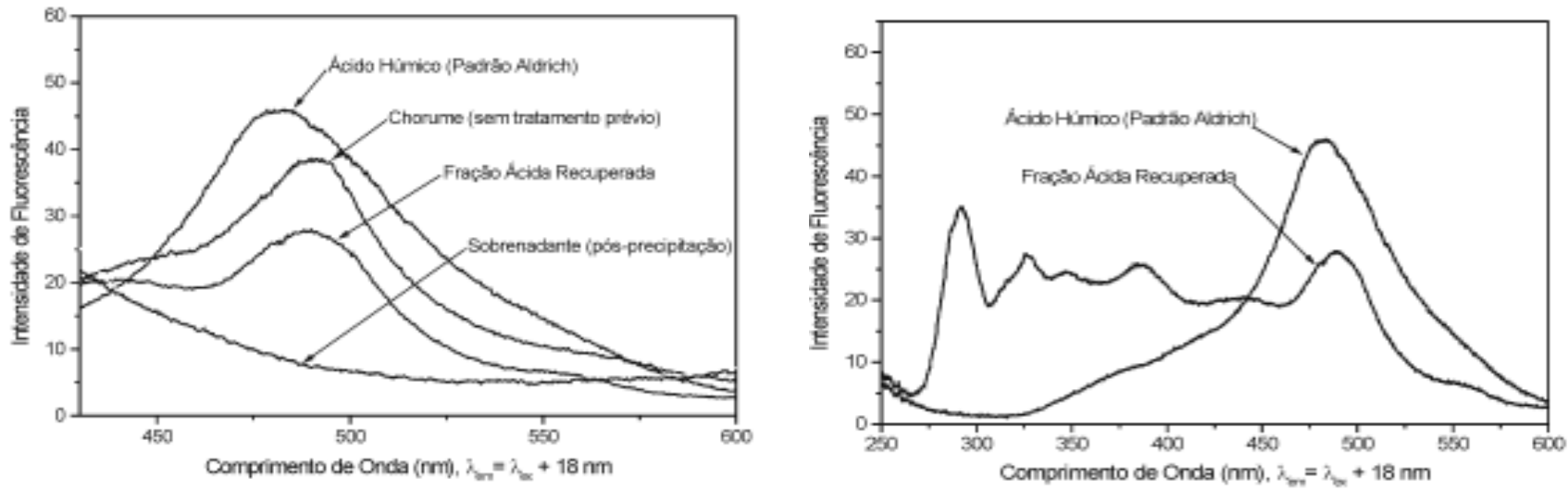

A

Figura 2 - Fluorescência em modo sincronizado para amostras de chorume e ácido húmico

Tabela 3 - Comparação dos processos oxidativos avançados no tratamento de chorume pré-tratado

\begin{tabular}{cccc}
\hline Processo & Redução da Área & Redução da DQO & Peróxido Residual \\
\hline $\mathrm{UV} / \mathrm{H}_{2} \mathrm{O}_{2}:$ & $70 \%$ & $53 \%$ & Ausente \\
$\mathrm{pH}=1,0 ;\left[\mathrm{H}_{2} \mathrm{O}_{2}\right]=1000 \mathrm{mg} \mathrm{L}^{-1}$ & & \\
Fenton (escuro): & $43 \%$ & $35 \%$ & $40 \%$ \\
$\mathrm{pH}=1,0 ;[\mathrm{Fe}]=5 \mathrm{mg} \mathrm{L}^{-1} ;\left[\mathrm{H}_{2} \mathrm{O}_{2}\right]=600 \mathrm{mg} \mathrm{L}^{-1}$ & & & $21 \%$ \\
$\mathrm{pH}=1,0 ;[\mathrm{Fe}]=5 \mathrm{mg} \mathrm{L}^{-1} ;\left[\mathrm{H}_{2} \mathrm{O}_{2}\right]=600 \mathrm{mg} \mathrm{L}^{-1}$ & $55 \%$ & $35 \%$ & \\
\hline
\end{tabular}



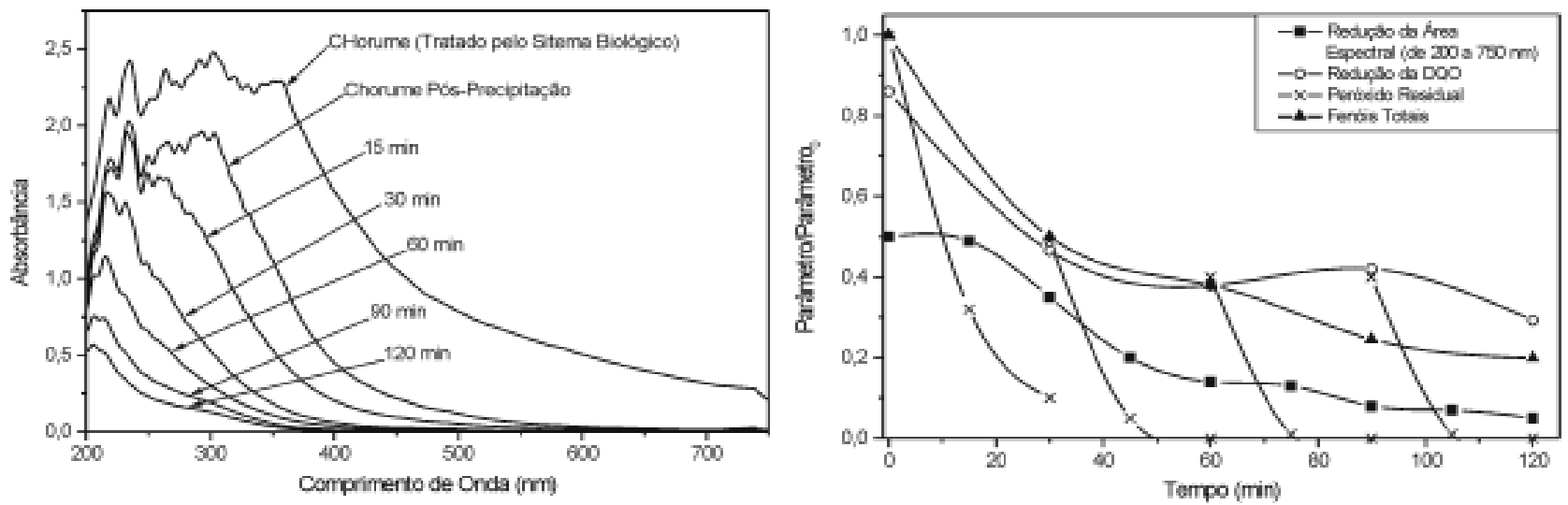

Figura 3 - Degradação de chorume por sistema UV/ $\mathrm{H}_{2} \mathrm{O}_{2}$ em sistemas de adições sucessivas. Sequência de espectros deUV-Vis (A) e evolução dos parâmetros monitorados (B)

Tabela 4 - resultados do estudo preliminar de tratamento para o processo UV/H O e sistema de adição contínua

\begin{tabular}{cccc}
\hline Processo & Redução DQO & $\begin{array}{c}\text { Redução da Área } \\
\text { espectral }\end{array}$ & $\mathrm{H}_{2} \mathrm{O}_{2}$ residual \\
\hline 3 vezes de $500 \mathrm{mg} \mathrm{L}^{-1}$ (0, 40 e $\left.80 \mathrm{~min}\right)$ & $50 \%$ & $95 \%$ & Ausente \\
5 vezes de $300 \mathrm{mg} \mathrm{L}^{-1}(0,20,40,60$ e $80 \mathrm{~min})$ & $63 \%$ & $93 \%$ & Ausente \\
8 vezes de $200 \mathrm{mg} \mathrm{L}^{-1}(0,10,20,30,40,50,60,70 \mathrm{~min})$ & $67 \%$ & $88 \%$ & Ausente \\
$1000 \mathrm{mg} \mathrm{L}^{-1}$ inicias + 2x300 mg L-1 (40 e $\left.80 \mathrm{~min}\right)$ & $71 \%$ & $96 \%$ & Ausente \\
$1000 \mathrm{mg} \mathrm{L}^{-1}$ inicias $+3 \times 200 \mathrm{mg} \mathrm{L}^{-1}(30,60$ e $90 \mathrm{~min})$ & $75 \%$ & $97 \%$ & Ausente \\
\hline
\end{tabular}

\section{CONCLUSÕES}

Quando aplicados de maneira isolada, os processos oxidativos avançados não apresentam boa eficiência, em razão de certas características do chorume serem incompatíveis com este tipo de processo. Dentre outras, contam-se: elevada carga orgânica e forte coloração.

O Processo de precipitação em meio ácido permite uma eficiente remoção de substâncias húmicas, carboidratos e outros compostos orgânicos, e que faz com que a cor do sistema diminua drasticamente.

Dentre todos os processos estudados, especial destaque para a precipitação seguida de tratamento por sistema $\mathrm{UV} / \mathrm{H}_{2} \mathrm{O}_{2}$. Quando no modo de adição contínua de peróxido, o sistema permitiu descoloraçôes da ordem de $96 \%$ e reduçóes de DQO de aproximadamente $75 \%$. Trata-se de um resultado bastante promissor, principalmente levando-se em consideração a elevada resistência da matriz em estudo.

\section{AGRADECIMENTOS}

À CAPES, CNPq e à Fundação Araucária pelo financiamento à pesquisa

\section{REFERÊNCIAS}

AGUER, J.-P.; RICHARD, C. Reatives species produced on irradiation at $365 \mathrm{~nm}$ of aqueous solutions of humic acids, Journal of photochemical and Photobiology A: Chemistry, n. 93, p. 193 - 198, 1996

APHA, Standard Methods for the Examination of Water and Wastewater. American Public Health Association, $19^{\text {th }}$ Edition, Washington DC, 1995.

BAE, B. et al. Treatment of Landfill Leachate Using Actived Sludge Process and Electro-Bean Radiation. Water Research. v. 33, n. 11, p. $2669-2673,1999$.

CAMEL, V.; BERMOND, A. The use of Ozone and Associated Oxidation Processes in Drinking Water Treatment. Water Research. v. 32, n. 11, p. $3208-3222,1998$.

CHAMARRO, E.; MARCO, A.; ESPULGAS, S. Use of Fenton Reagent to improve organic Chemical Biodegradability. Water Research. Vol. 35, n. 4, p. $1047-1051,2001$.
CHEN, J. et al. Fluorescence spectroscopic studies of natural organic matter fraction. Chemosphere. v. 50 , p. $639-647,2003$

CHRISTENSEN, T. et al. Biogeochemistry of Landfill Leachate Plumes. Applied Geochemistry, v. 16, p. $659-718,2001$.

CRITENDEN, J. C. et al. A kinect model for $\mathrm{H}_{2} \mathrm{O} J U V$ process in a completely mixed batch reactor. Water Research. v. 33, n. 10, p $2315-$ 2328, 2001.

ESPULGAS, S. et al. Comparison of different advanced oxidation processes for phenol degradation. Water Research. v. 36, p. $1034-$ 1042,2002 .

FREIRE, R. S. et al. P. Novas tendências para o tratamento de resíduos industriais contendo espécies organocloradas. Química Nova. v. 23, n. 4, p. $504-511,2000$.

GHALY, M. Y. el al. Photochemical Oxidation of p-chlorophenol by $\mathrm{UV}_{\mathrm{H}} \mathrm{H}_{2} \mathrm{O}_{2}$ and Photo-Fenton process. A Comparative study. Waste Management. v. 21. p. $41-47,2001$.

http://www.ibge.gov.br (acessado em 18 de dezembro de 2003)

IM, J. H. et al. Simultaneous Organic and Nitrogen Removal from Municipal Landfill Leachate Using an Anaerobic-Aerobic Sistem. Water Research. v. 35, n. 10, p. $2403-2410,2001$. 
JARDIM, W. F. et al. Short-team toxicity test using Escherichia coli: Monitoring $\mathrm{CO}_{2}$ production by flow injection analysis. Water Research. v. 24 , p. $351-354,1990$.

KANG, K.-H.; SHIN, H. S.; PARK, H. Characterization of humic substances present in a landfill leachate with different landfill ages and its applications. Water Researh. n. 26, p. 4023 - 4032, 2002.

KUNZ, A. et al. Novas tendências para o tratamento de Efluentes têxteis. Química Nova. v. 25 , n. 1 , p. $78-82,2002$

LEAHY, J. G., SHREVE, G. S. The effect of Organic Carbon on the Sequential Reductive Dehalogenation of Tetrachoethylene in Landfill Leachates. Water Research. v. 34, n. 8, p. 2390 - 2396. 2000.

LIN, S. H. CHANG, C. C. Treatament of Landfill Leachate by Combined Electro-Fenton Oxidation and Sequencing Batch Reactor Method. Water Research. v. 34, n. 17, p. $4243-4249$, 2000 .

LO, I. M. Characteristics and Treatament of Leachates From Domestic Landfills. Environment International. v. 22 n. 4, p. $433-442,1996$.
NANNY, M. A. RATASUK, N. Characterization and comparison of hydrophobic neutral and hydrophobic acid dissolved organic carbon isolated from three municipal landfill leachate. Water Research. v. 36, p. 1572 - 1584, 2002.

NEYENS, E.; BAYENS, J. A review of classic Fenton's peroxidation as an advanced technique. Journal of Hazardous Materials. v. 98, p. 33 50, 2003.

OLIVEIRA, M. C. et al. Sistema de injeção em fluxo espectrofotométrico para monitorar peróxido de hidrogênio residual em processo de fotodegradação por reação foto-Fenton. Quimica Nova. v. 24, n. 2, p. 188 - 190, 2001

PEURAVUORI, J.; KOIVIKKO, R.; PIHLAJA, K. Characterization, differentiation and classification of aquatic humic matter separated with different sorbents: synchronous scanning fluorescence spectroscopy. Water Research. v. 36, p. 4552 - 4562, 2002.

STROOT, P. G. et al. Anaerobic Condigestion of Municipal, Solid Waste and Biosolids Under Various Mixing Conditions - II. Microbial Population Dynamics. Water Research. v. 35, n. 7, p. 1817 - 1827, 2001.
WU, K. et al. Photo-Fenton degradation of a dye under visible light irradiation. Journal of Molecular Catalysis A: Chemical. n.144. p. 77 - 84. 1999 .

YOON, J.; CHO, Y.; KIM, S. The characteristics of Coagulation of Fenton Reaction in the Removal of Landfill Leachate Organics. Water Science and Technology, v. 38, n. 2, 1998.

\section{Endereço para correspondência:}

Patrício G. Peralta-Zamora Universidade Federal do Paraná Departamento de Química Cx.Postal: 1908I CEP: 8I53I-990 Curitiba - PR Brasil

E-mail: zamora@quimica.ufpr.br

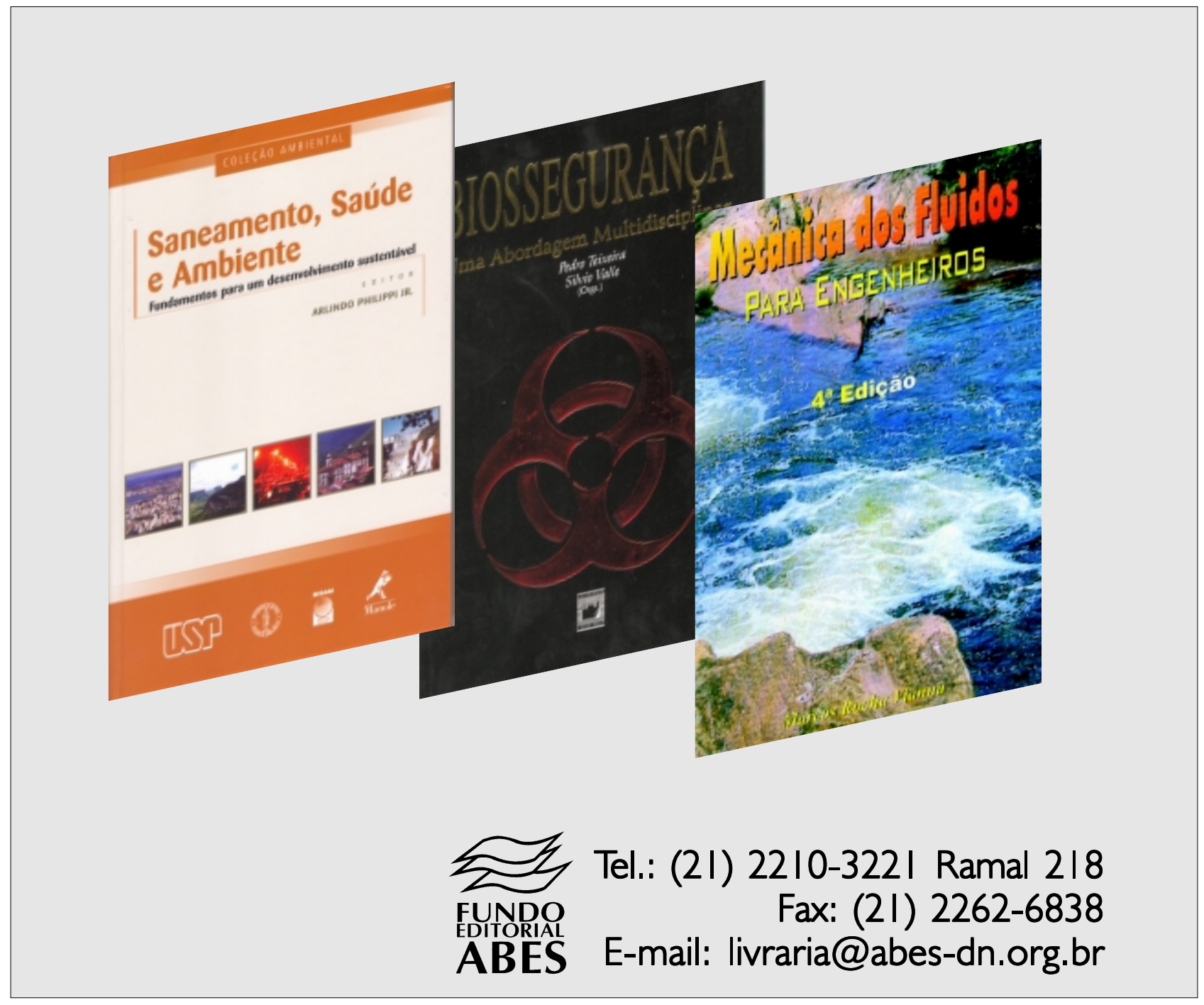

